\title{
Predictor-Based Model Reference Adaptive Control
}

\author{
Eugene Lavretsky ${ }^{1}$, Ross Gadient ${ }^{2}$, and Irene M. Gregory ${ }^{3}$ \\ The Boeing Company, Huntington Beach, CA 92647, USA, and NASA Langley Research Center, Hampton, VA \\ 23681-2199, USA
}

\begin{abstract}
This paper is devoted to robust, Predictor-based Model Reference Adaptive Control (PMRAC) design. The proposed adaptive system is compared with the now-classical Model Reference Adaptive Control (MRAC) architecture. Simulation examples are presented. Numerical evidence indicates that the proposed PMRAC tracking architecture has better than MRAC transient characteristics.
\end{abstract}

\section{Nomenclature}

$A_{p}, B_{p}, C_{p}=$ original system matrices

$A, B, B_{c}, C=$ extended system matrices

$A_{\text {ref, }} A_{\text {prd }}=$ Hurwitz matrices for reference model and predictor

$\Lambda \quad=$ unknown diagonal matrix, with positive elements

$u \quad=$ control input vector

$x_{p}, x=$ system state vectors

$y=$ controlled system output vector

$n_{p}, n, m=$ state and control dimensions

$d(x) \quad=$ matched uncertainty

$\Phi_{d}\left(x_{p}\right)=$ known regressor vector

$N \quad=$ dimension of the regressor vector

$\Theta_{D} \quad=$ matrix of constant unknown parameters

$r(t) \quad=$ external bounded time-varying vector of commands

\section{Introduction}

$\mathrm{T}$ his paper is devoted to the design and analysis of a state-predictor based direct adaptive controller for MultiInput-Multi-Output (MIMO) dynamical systems, with matched uncertainties. Our main intent is to evaluate possibility of improving MRAC transient characteristics during a tracking task. Towards that end, we propose the use of a state-predictor in formulating adaptive laws. Specifically, starting with a direct MRAC system, [1, 2, 4], we design a state predictor, while using the system full state measurements. Then through Lyapunov' stability analysis, we propose a predictor-based modification to the MRAC laws. This modification constitutes the novelty of the results that are reported here.

The rest of the paper is organized as follows. In Section II, MIMO system dynamics are defined and the control goal is formulated. In section III, predictor-based MRAC laws are derived, and stability proof is conducted using conventional (in adaptive control) Lyapunov-based arguments. Simulation example is presented in Section IV, followed by conclusions that are given in Section V.

\section{Model Definition}

In this section, we formulate the system dynamics, pose the control problem, and derive tracking error dynamics for adaptive control design. We begin by considering a class of MIMO uncertain systems in the form:

\footnotetext{
${ }^{1}$ Sr. Technical Fellow, The Boeing Company, AIAA Assoc. Fellow.

${ }^{2}$ Engineer, The Boeing Company, AIAA Member.

${ }^{3}$ Sr. Research Engineer, NASA Langley Research Center, AIAA Sr. Member.
} 


$$
\dot{x}_{p}=A_{p} x_{p}+B_{p} \Lambda(u+\overbrace{\Theta_{d}^{T} \Phi_{d}\left(x_{p}\right)}^{d\left(x_{p}\right)})
$$

where $x_{p} \in X_{p} \subset R^{n_{p}}$ is the system state vector, $u \in U \subset R^{m}$ is the control input,

$$
d\left(x_{p}\right)=\Theta_{d}^{T} \Phi_{d}\left(x_{p}\right)
$$

is the linear-in-parameters state-dependent matched uncertainty, $\Theta_{d} \in R^{N \times m}$ is the matrix of unknown constant parameters, and $\Phi_{d}\left(x_{p}\right) \in R^{N}$ is the known $N$ - dimensional regressor vector, whose components are piece-wise continuous functions of $x_{p}$. Also in (2.1), $B_{p} \in R^{n \times m}$ is constant and known, $A_{p} \in R^{n \times n}$ is constant and unknown, and $\Lambda \in R^{m \times m}$ is constant, diagonal and unknown matrix with positive diagonal elements.

The control goal of interest is bounded tracking, that is one needs to define $u$ such that the system controlled output

$$
y=C_{p} x_{p} \in R^{m}
$$

tracks any bounded and possibly time-varying command $r(t) \in R^{m}$, with bounded errors, in the presence of the system uncertainties $\left\{A_{p}, \Lambda, \Theta_{d}\right\}$.

Let

$$
e_{y}(t)=y(t)-r(t)
$$

denote the system output tracking error. Augmenting (2.1) with the integrated output tracking error,

$$
\left(e_{y I}(t)=\int_{0}^{t} e_{y}(\tau) d \tau\right) \Leftrightarrow\left(e_{y I}=\frac{e_{y}}{s}\right)
$$

yields extended open-loop system dynamics:

$$
\dot{x}=A x+B \Lambda\left(u+d\left(x_{p}\right)\right)+B_{c} r
$$

where $x=\left(\begin{array}{ll}e_{y I}^{T} & x_{p}^{T}\end{array}\right) \in X \subset R^{n}$ is the extended system state vector, whose dimension is $n=n_{p}+m$. The system matrices $\left(A, B, B_{c}\right)$ are

and

$$
A=\left(\begin{array}{ll}
0_{m \times m} & C_{p} \\
0_{n_{p} \times m} & A_{p}
\end{array}\right), B=\left(\begin{array}{c}
0_{m \times m} \\
B_{p}
\end{array}\right), B_{c}=\left(\begin{array}{c}
-I_{m \times m} \\
0_{n_{p} \times m}
\end{array}\right)
$$

represents the system controlled output. To summarize, in this paper we will consider MIMO uncertain systems in the form of (2.6):

$$
\left\{\begin{array}{l}
\dot{x}(t)=A x(t)+B \Lambda\left(u+\Theta_{d}^{T} \Phi_{d}\left(x_{p}\right)\right)+B_{c} r(t) \\
y(t)=C x(t)
\end{array}\right.
$$

with an unknown constant matrix $A \in R^{n \times n}$, known matrices $B \in R^{n \times m}, B_{c} \in R^{n \times m}, C \in R^{m \times n}$, an unknown constant diagonal positive-definite matrix $\Lambda \in R^{m \times m}$, and an unknown matrix of constant parameters $\Theta_{d} \in R^{N \times m}$. The statedependent regressor vector $\Phi_{d}\left(x_{p}\right) \in R^{N}$ is assumed to be known. The control goal is bounded tracking of any bounded time-varying reference signal $r(t) \in R^{m \times 1}$ by the system output $y(t) \in R^{m \times 1}$, in the presence of constant parametric uncertainties $\left(A, \Lambda, \Theta_{d}\right)$.

\section{Assumption 2.1 (Model Matching Conditions)}


Given a reference Hurwitz matrix $A_{\text {ref }}$ and an unknown positive-definite diagonal constant matrix $\Lambda$, there must exist a constant possibly unknown gain matrix $K_{x}$ such that

$$
A_{r e f}=A+B \Lambda K_{x}^{T}
$$

Using the assumed relation (2.10), we rewrite the system dynamics in the form:

$$
\dot{x}=A_{r e f} x+B \Lambda\left(u-K_{x}^{T} x+\Theta_{d}^{T} \Phi_{d}\left(x_{p}\right)\right)+B_{c} r
$$

Control input $u$ is chosen as:

$$
u=\hat{K}_{x}^{T} x-\hat{\Theta}_{d}^{T} \Phi_{d}\left(x_{p}\right)
$$

where $\hat{K}_{x}(t) \in R^{n \times m}$ and $\hat{\Theta}_{d}(t) \in R^{N \times m}$ are adaptive time-varying matrices, whose dynamics will be defined later. Substituting (2.12) into (2.11), yields:

$$
\begin{aligned}
\dot{x} & =A_{\text {ref }} x+B \Lambda(\underbrace{\left(\hat{K}_{x}-K_{x}\right)^{T} x}_{\Delta K_{x}}-(\underbrace{\left.\hat{\Theta}_{d}-\Theta_{d}\right)^{T} \Phi_{d}}_{\Delta \Theta_{d}}\left(x_{p}\right))+B_{c} r \\
& =A_{\text {ref }} x+B \Lambda\left(\Delta K_{x}^{T} x-\Delta \Theta_{d}^{T} \Phi_{d}\left(x_{p}\right)\right)+B_{c} r
\end{aligned}
$$

Based on (2.13), consider the following reference model:

and let

$$
\dot{x}_{\text {ref }}=A_{\text {ref }} x_{\text {ref }}+B_{c} r
$$

$$
e=x-x_{\text {ref }}
$$

represent the system tracking error. Subtracting (2.14) from (2.13) results in the tracking error dynamics,

$$
\dot{e}=A_{r e f} e+B \Lambda\left(\Delta K_{x}^{T} x-\Delta \Theta_{d}^{T} \Phi_{d}\left(x_{p}\right)\right)
$$

\section{Remark 2.1}

Based on (2.16) and using Lyapunov arguments, direct Model Reference Adaptive Control (MRAC) laws can be derived [1], [2] in the form

$$
\left\{\begin{array}{l}
\dot{\hat{K}}_{x}=-\Gamma_{x} x e^{T} P_{r e f} B \\
\dot{\hat{\Theta}}_{d}=\Gamma_{\Theta_{d}} \Phi_{d} e^{T} P_{r e f} B
\end{array}\right.
$$

where $P_{r e f}=P_{r e f}^{T}>0$ is the unique symmetric positive definite solution of the algebraic Lyapunov equation,

$$
A_{\text {ref }}^{T} P_{\text {ref }}+P_{\text {ref }} A_{\text {ref }}=-Q_{\text {ref }}
$$

and $Q_{\text {ref }}=Q_{r e f}^{T}>0$. Relations (2.12) and (2.17) solve the tracking problem, with globally asymptotically stable closed-loop dynamics. This particular solution is valid for any symmetric positive definite rates of adaptation $\Gamma_{x}$ and $\Gamma_{\boldsymbol{\theta}_{d}}$. However, if these matrices have large singular values, then unwanted transient oscillations will often occur in the system.

\section{Predictor-Based MRAC}

In this section, a PMRAC-based solution to servomechanism problem is presented, for the class of uncertain systems in the form of (2.9). Towards that end and based on (2.11), define the so-called predictor dynamics:

$$
\dot{\hat{x}}=A_{p r d}(\hat{x}-x)+A_{r e f} x+B_{c} r
$$

where $A_{p r d}$ is a Hurwitz matrix.

Using relation (2.15), let

$$
\left\{\begin{array}{l}
e=x-x_{\text {ref }} \\
\hat{e}=\hat{x}-x
\end{array}\right.
$$

represent the tracking and prediction errors, respectively. Subtracting (2.14) from (2.13) and (2.13) from (3.1), results in the tracking and prediction error dynamics. 


$$
\left\{\begin{array}{l}
\dot{e}=A_{r e f} e+B \Lambda\left(\Delta K_{x}^{T} x-\Delta \Theta_{d}^{T} \Phi_{d}\left(x_{p}\right)\right) \\
\dot{\hat{e}}=A_{p r d} \hat{e}-B \Lambda\left(\Delta K_{x}^{T} x-\Delta \Theta_{d}^{T} \Phi_{d}\left(x_{p}\right)\right)
\end{array}\right.
$$

Our design approach is Lyapunov based, and because of that in order to derive stable adaptive laws, consider the following Lyapunov function candidate:

$$
V\left(e, \hat{e}, \Delta K_{x}, \Delta \Theta_{d}\right)=e^{T} P_{r e f} e+\hat{e}^{T} P_{p r d} \hat{e}+\operatorname{trace}\left(\Delta K_{x}^{T} \Gamma_{x}^{-1} \Delta K_{x} \Lambda\right)+\operatorname{trace}\left(\Delta \Theta_{d}^{T} \Gamma_{\Theta_{d}}^{-1} \Delta \Theta_{d} \Lambda\right)
$$

where $\Gamma_{x}=\Gamma_{x}^{T}>0$ and $\Gamma_{\Theta_{d}}=\Gamma_{\theta_{d}}^{T}>0$ denote constant rates of adaptation, while $P_{r e f}=P_{r e f}^{T}>0$ and $P_{p r d}=P_{p r d}^{T}>0$ are the two unique solutions of the two algebraic Lyapunov equations, correspondingly.

$$
\left\{\begin{array}{l}
A_{r e f}^{T} P_{r e f}+P_{r e f} A_{r e f}=-Q_{r e f} \\
A_{p r d}^{T} P_{p r d}+P_{p r d} A_{p r d}=-Q_{p r d}
\end{array}\right.
$$

with $Q_{r e f}=Q_{r e f}^{T}>0$ and $Q_{p r d}=Q_{p r d}^{T}>0$. Time derivative of the Lyapunov function $V\left(e, \hat{e}, \Delta K_{x}, \Delta \Theta_{d}\right)$, evaluated along trajectories of the error dynamics (3.3), yields

$$
\begin{aligned}
& \dot{V}=-e^{T} Q_{r e f} e-\hat{e}^{T} Q_{p r d} \hat{e}+2\left(e^{T} P_{r e f}-\hat{e}^{T} P_{p r d}\right) B \Lambda\left(\Delta K_{x}^{T} x-\Delta \Theta_{d}^{T} \Phi_{d}\left(x_{p}\right)\right) \\
& +2 \operatorname{trace}\left(\Delta K_{x}^{T} \Gamma_{x}^{-1} \dot{\hat{K}}_{x} \Lambda\right)+2 \operatorname{trace}\left(\Delta \Theta_{d}^{T} \Gamma_{\Theta_{d}}^{-1} \dot{\hat{\Theta}}_{d} \Lambda\right)
\end{aligned}
$$

Define the training error signal:

$$
\bar{e}=B^{T}\left(P_{r e f} e-P_{p r d} \hat{e}\right)
$$

Applying the well-known trace identity (valid for any two co-dimensional vectors $a$ and $b$ ),

$$
a^{T} b=\operatorname{trace}\left(b a^{T}\right)
$$

the time derivative in (3.6) becomes:

$$
\begin{aligned}
& \dot{V}=-e^{T} Q_{r e f} e-\hat{e}^{T} Q_{p r d} \hat{e} \\
& +2 \operatorname{trace}\left(\Delta K_{x}^{T} x \bar{e}^{T} \Lambda\right)+2 \operatorname{trace}\left(\Delta K_{x}^{T} \Gamma_{x}^{-1} \dot{\hat{K}}_{x} \Lambda\right)-2 \operatorname{trace}\left(\Delta \Theta_{d}^{T} \Phi_{d}\left(x_{p}\right) \bar{e}^{T} \Lambda\right)+2 \operatorname{trace}\left(\Delta \Theta_{d}^{T} \Gamma_{\Theta_{d}}^{-1} \dot{\hat{\Theta}}_{d} \Lambda\right)
\end{aligned}
$$

Collecting similar terms, yields

$$
\begin{aligned}
& \dot{V}=-e^{T} Q_{r e f} e-\hat{e}^{T} Q_{p r d} \hat{e} \\
& +2 \operatorname{trace}\left(\Delta K_{x}^{T}\left\{{ }_{x}^{-1} \dot{\hat{K}}_{x}+x \bar{e}^{T}\right\}\right)+2 \operatorname{trace}\left(\Delta \Theta_{d}^{T}\left\{\int_{\Theta_{d}}^{-1} \dot{\hat{\Theta}}_{d}-\Phi_{d}\left(x_{p}\right) \bar{e}^{T}\right\}\right)
\end{aligned}
$$

Consequently, choosing the following PMRAC laws,

implies that

$$
\left\{\begin{array}{l}
\dot{\hat{K}}_{x}=-\Gamma_{x} x \bar{e}^{T} \\
\dot{\hat{\Theta}}_{d}=\Gamma_{\Theta_{d}} \Phi_{d}\left(x_{p}\right) \bar{e}^{T}
\end{array}\right.
$$

which in turn, proves Uniform Ultimate Boundedness (UUB) [3] of $\left(e, \hat{e}, \Delta K_{x}, \Delta \Theta_{d}\right)$. Moreover, both the tracking and prediction errors are square integrable: $e, \hat{e} \in L_{2}$. Furthermore, since $r \in L_{\infty}$ then $x_{r e f} \in L_{\infty}$, then consequently, $x \in L_{\infty}$. This implies $\hat{x} \in L_{\infty}$. Since ideal parameters $\left(K_{x}, \Theta_{d}\right)$ are constant and their estimation errors $\left(\Delta K_{x}, \Delta \Theta_{d}\right)$ are bounded, then their estimated values are bounded as well, that is: $\hat{K}_{x}, \hat{\Theta}_{d} \in L_{\infty}$. Since components of the regressor vector $\Phi_{d}\left(x_{p}\right)$ are piece-wise continuous functions of $x_{p}$ and the latter is bounded, the components themselves are bounded. Hence, $u \in L_{\infty}$ and $\dot{x}, \dot{\hat{x}} \in L_{\infty}$. Thus $\dot{e}, \dot{\hat{e}} \in L_{\infty}$ which implies that $\ddot{V} \in L_{\infty}$. Therefore, $\dot{V}$ is a uniformly continuous function of time. Since $V$ is lower bounded, $\dot{V} \leq 0$, and $\dot{V}$ is uniformly continuous then $V$ tends to a limit, while its derivative $\dot{V}$ tends to zero. This fact is known as Barbalat's Lemma, [1], [2], [3]. Consequently, the tracking and prediction errors, $e$ and $\hat{e}$, tend to zero asymptotically, as $t \rightarrow \infty$. Moreover, since Lyapunov function (3.4) is radially unbounded, the asymptotic convergence of the errors is global. 
In other words, the closed-loop tracking and prediction error dynamics are globally asymptotically stable. The tracking problem is solved. The end result is summarized below.

\section{Theorem 3.1}

Consider the uncertain system dynamics in (2.9), operating under PMRAC controller (3.11), with the state predictor (3.1). Suppose that the matching relations (2.10) hold. Let the reference model (2.14) be driven by some bounded and possibly time-varying reference command $r(t)$. Then:

a) All signals in the closed-loop system,

$$
\left\{\begin{array}{l}
\dot{x}=A x+B \Lambda\left(\hat{K}_{x}^{T} x-\hat{\Theta}_{d}^{T} \Phi_{d}\left(x_{p}\right)+\Theta_{d}^{T} \Phi_{d}\left(x_{p}\right)\right)+B_{c} r(t) \\
\dot{x}_{r e f}=A_{r e f} x_{r e f}+B_{c} r(t) \\
\dot{\hat{x}}=A_{p r d}(\hat{x}-x)+A_{r e f} x+B_{c} r(t) \\
\dot{\hat{K}}_{x}=-\Gamma_{x} x\left(\left(x-x_{r e f}\right)^{T} P_{r e f}-(\hat{x}-x)^{T} P_{p r d}\right) B \\
\dot{\hat{\Theta}}_{d}=\Gamma_{\mathbf{\theta}_{d}} \Phi_{d}\left(x_{p}\right)\left(\left(x-x_{r e f}\right)^{T} P_{r e f}-(\hat{x}-x)^{T} P_{p r d}\right)
\end{array}\right.
$$

are uniformly bounded in time, where symmetric positive definite matrices $P_{r e f}$ and $P_{p r d}$ are the two unique solutions of the two algebraic Lyapunov equations in (3.5), respectively.

b) Tracking error $e=x-x_{\text {ref }}$ is UUB, square integrable, and globally asymptotically stable, $\lim _{t \rightarrow \infty}\|e(t)\|=0$.

c) Prediction error $\hat{e}=\hat{x}-x$ is UUB, square integrable, and globally asymptotically stable, $\lim _{t \rightarrow \infty}\|\hat{e}(t)\|=0$.

\section{Remark 3.1}

The developed PMRAC design methodology can be applied to the original system (2.1), and without adding integrated output tracking errors. In this case, the control input is:

$$
u=\hat{K}_{x}^{T} x+\hat{K}_{r}^{T} r-\hat{\Theta}_{d}^{T} \Phi_{d}\left(x_{p}\right)
$$

where $\hat{K}_{r} \in R^{m \times m}$ is the estimated feedforward gain. The system closed-loop dynamics become:

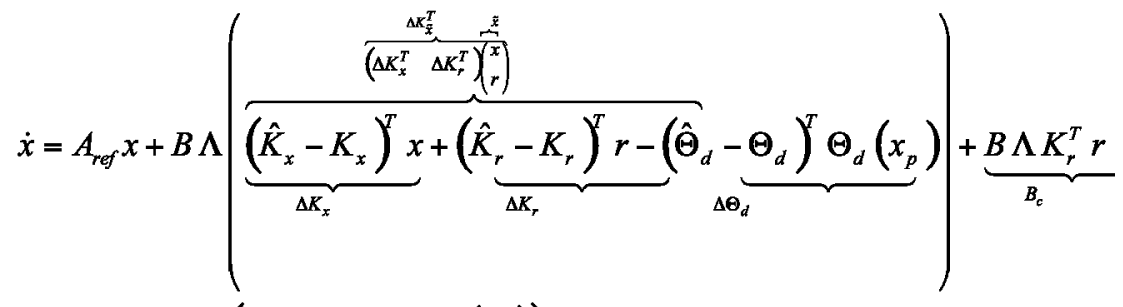

$$
\begin{aligned}
& =A_{\text {ref }} x+B \Lambda\left(\Delta K_{\tilde{x}}^{T} \tilde{x}-\Delta \Theta_{d}^{T} \Phi_{d}\left(x_{p}\right)\right)+B_{c} r
\end{aligned}
$$

where $K_{r} \in R^{m \times m}$ is the unknown / ideal feedforward gain. The relation $B_{c}=B \Lambda K_{r}^{T}$ is the required matching condition which guarantees existence of the ideal feedforward gain. The corresponding PMRAC laws are:

$$
\begin{aligned}
& \dot{\hat{K}}_{x}=-\Gamma_{x} x\left(\left(x-x_{r e f}\right)^{T} P_{r e f}-(\hat{x}-x)^{T} P_{p r d}\right) B \\
& \dot{\hat{K}}_{r}=-\Gamma_{r} r\left(\left(x-x_{r e f}\right)^{T} P_{r e f}-(\hat{x}-x)^{T} P_{p r d}\right) B \\
& \dot{\hat{\Theta}}_{d}=\Gamma_{\Theta_{d}} \Phi_{d}\left(x_{p}\right)\left(\left(x-x_{r e f}\right)^{T} P_{r e f}-(\hat{x}-x)^{T} P_{p r d}\right)
\end{aligned}
$$

where $\Gamma_{r}=\Gamma_{r}^{T}>0$ is the adaptation rate matrix for the feedforward adaptive gain $\hat{K}_{r}$.

\section{Remark 3.2}

Comparing MRAC laws (2.17) with PMRAC laws (3.11), it is evident that the presence of the state predictor (3.1) adds the low-pass filtering effects of the prediction dynamics to the direct MRAC laws. 


\section{Remark 3.3}

PMRAC design extension to MIMO systems with non-parametric uncertainties is straightforward and can be accomplished by using well-known in adaptive control robustification methods [1], [2], [4], [5], such as: a) Deadzone, b) $\sigma$ - modification, c) e-modification, and d) Projection Operator.

\section{Simulation Example}

In this section, we will demonstrate PMRAC benefits using a simplified model which is representative of aircraft dynamics. Through simulation evaluations, we compare closed-loop tracking performance of the proposed PMRAC and the "classical" MRAC controllers. Our focus is on the transient performance of these two controllers. We show that in transient, PMRAC yields less oscillations than MRAC. We also show that PMRAC adaptation rates can be increased substantially and without inducing unwanted oscillations that would otherwise be present if using MRAC. We immediately note that these claims about transient behavior represent numerical observations only. Efforts to obtain formal quantification of transient dynamics provided by these two adaptive controllers constitute an ongoing research and will be reported at a later time.

Our simulation model is chosen to represent longitudinal dynamics of an aerial vehicle, such as an F-16 aircraft. Neglecting the effects of gravity and thrust, aircraft longitudinal (short period) dynamics can be written in matrix form [6],

$$
\underbrace{\left(\begin{array}{c}
\dot{\alpha} \\
\dot{q}
\end{array}\right)}_{\dot{x}_{p}}=\underbrace{\left(\begin{array}{cc}
\frac{Z_{\alpha}}{V} & 1+\frac{Z_{q}}{V} \\
M_{\alpha} & M_{q}
\end{array}\right)}_{A_{p A L}} \underbrace{\left(\begin{array}{c}
\alpha \\
q
\end{array}\right)}_{x_{p}}+\underbrace{\left(\begin{array}{c}
\frac{Z_{\delta}}{V} \\
M_{\delta_{e}}
\end{array}\right)}_{B_{p}} \underbrace{\delta_{e}}_{u}
$$

where $\alpha$ is the aircraft angle of attack (AOA), $q$ is the pitch rate, $\delta_{e}$ is the elevator deflection (control input), $V$ is the trimmed (constant) airspeed, $\left(Z_{\alpha}, Z_{q}, Z_{\delta_{e}}\right)$ and $\left(M_{\alpha}, M_{q}, M_{\delta_{e}}\right)$ are partial derivatives of the aerodynamic vertical force $Z$ and the pitching moment $M$, with respect to $\left(\alpha, q, \delta_{e}\right)$ respectively. Numerical values for the vehicle aerodynamic derivatives were taken from [6, Example 5.5-3, Table 3.4-3]. These data represent an F-16 aircraft trimmed at:

$$
V_{T}=502 f t \mathrm{sec}, \quad A l t=0 \mathrm{f}, t^{-} \quad \mathcal{Q} 300 \quad l b \stackrel{f}{\mathrm{f}, t} \quad C \in 0.35^{-}, c \alpha=2.11 \mathrm{deg}
$$

The resulting open-loop system matrices are:

$$
A_{p B L}=\left(\begin{array}{cc}
-1.0189 & 0.9051 \\
0.8223 & -1.0774
\end{array}\right), \quad B_{p}=\left(\begin{array}{c}
-0.0022 \\
-0.1756
\end{array}\right)
$$

where $\alpha$ is in radians, $q$ is in radians / second, and $\delta_{e}$ is in degrees.

Three types of matched uncertainties are added to the system: a) linear-in-state uncertainty $K_{x_{p}}^{T}$ pert $x_{p}$, b) control effectiveness constant uncertainty $\Lambda>0$, and c) nonlinear-in-state uncertainty in the form of (2.2). Numerical values for the uncertainties were arbitrarily chosen as:

$$
K_{x_{p} \text { pert }}^{T}=(-4.6839-6.1373), \quad \Lambda=0.5, d\left(x_{p}\right)=d(\alpha)=0.5 e^{-\frac{\left(\alpha-\alpha_{c}\right)^{2}}{2 \sigma^{2}}}
$$

where the center of the Gaussian was set to $\alpha_{c}=2^{\circ} \frac{\pi}{180}$, and its width was $\sigma=0.0233$. This particular selection of numerical values for $K_{x_{p} \text { pert }}^{T} x_{p}$ and $\Lambda$ is equivalent to $50 \%$ increase in the static stability $M_{\alpha}, 50 \%$ decrease in the pitch damping $M_{q}$, and $50 \%$ decrease in the elevator effectiveness $M_{\delta_{e}}$. These changes imply that the vehicle became $50 \%$ more statically unstable, lost $50 \%$ of its pitch damping ability, and the aircraft controllability decreased by $50 \%$. Such drastic and perhaps unrealistic changes were motivated by our intent to demonstrate the effectiveness of the proposed PMRAC design methodology. 
The system total matched uncertainty versus AOA was calculated at $q=0$, and is shown in the figure below.

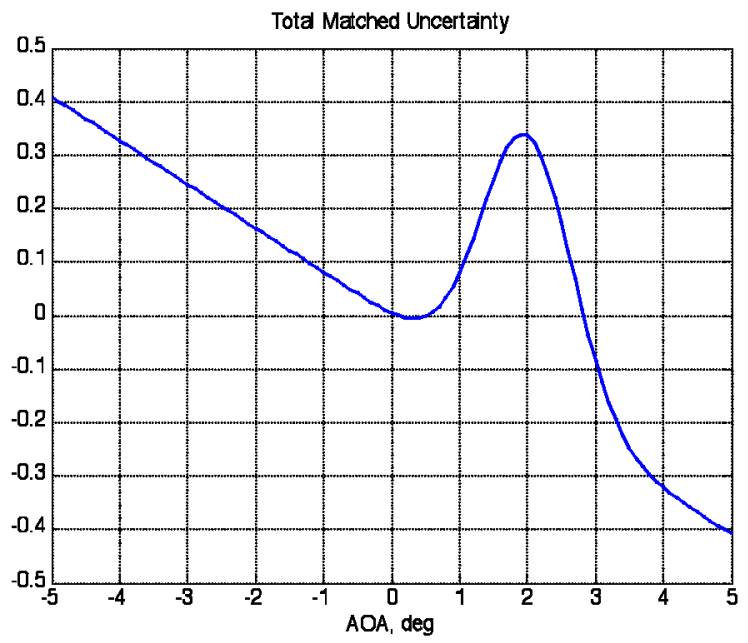

Figure 4.1: Total Matched Uncertainty versus AOA, at $q=0$

With all the uncertainties included, the aircraft dynamics take the form of (2.1),

$$
\dot{x}_{p}=\underbrace{\left(A_{p B L}+B_{p} \Lambda K_{x_{p} p e r t}^{T}\right) x_{p}}_{A_{p}}+B_{p} \Lambda\left(u+d\left(x_{p}\right)\right)
$$

where $x_{p}=\left(\begin{array}{ll}\alpha & q\end{array}\right)^{T}$ is the system state vector, $\Lambda>0$ is the uncertainty in the elevator effectiveness, and the statedependent function $d\left(x_{p}\right)$ represents unknown nonlinear increments in the vehicle aerodynamic $Z$-force and the pitching moment $M$. The vehicle pitch rate $q$ is selected to represent the system controlled output.

$$
y=q=\underbrace{\left(\begin{array}{ll}
0 & 1
\end{array}\right) x_{p}}_{C_{p}}=C_{p} x_{p}
$$

Thus, the control goal is to track any bounded time-varying pitch rate command,

$$
r=q_{\text {cmd }}(t)
$$

in the presence of the system uncertainties.

Adding integrated pitch rate tracking error dynamics,

$$
\dot{e}_{I}=q-q_{\text {cmd }}
$$

results in the extended open-loop dynamics, which is in the form of (2.9).

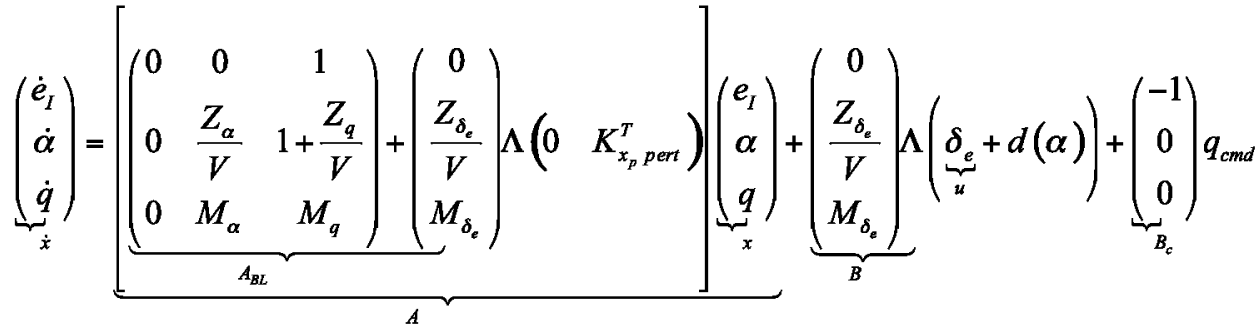

$$
\begin{aligned}
& \underbrace{q}_{y}=\underbrace{\left(\begin{array}{lll}
0 & 0 & 1
\end{array}\right) x}_{C}
\end{aligned}
$$

Conventional MRAC architecture was designed using adaptive laws (2.17). The regressor vector $\Phi_{d}(\alpha)$ consisted of $11 \alpha$-dependent and evenly spaced Gaussians (RBF-s). RBF centers were placed at $[-10: 2: 10]$ degrees of AOA, and all RBF widths were set to $\sigma=0.0233$. Reference matrix $A_{r e f}$ was defined using LQR method with 


$$
Q=\operatorname{diag}\left(\left[\begin{array}{lll}
200 & 0 & 0
\end{array}\right]\right), \quad R=1
$$

The corresponding baseline $L Q R$ feedback gains were calculated as:

$$
K_{x B L}^{T}=\left(\begin{array}{lll}
14.1421 & 3.3331 & 9.1263
\end{array}\right)
$$

Using the baseline LQR control feedback,

the reference model was formed with:

$$
\delta_{e B L}=K_{x B L}^{T} x
$$

$$
A_{\text {ref }}=A_{B L}+B K_{x B L}^{T}
$$

It is straightforward to verify that such a selection of $A_{\text {ref }}$ satisfies the matching conditions (2.10). The eigenvalues of the reference dynamics, along with their corresponding natural frequencies and damping ratios, are shown in the table below.

\begin{tabular}{|c|c|c|}
\hline $\begin{array}{c}\text { Eigenvalue } \\
\mathrm{n} / \mathrm{d}\end{array}$ & $\begin{array}{c}\text { Damping } \\
\mathrm{n} / \mathrm{d}\end{array}$ & $\begin{array}{c}\text { Natural Frequency } \\
\mathrm{rad} / \mathrm{sec}\end{array}$ \\
\hline$-1.12 \mathrm{e}+000+6.99 \mathrm{e}-001 \mathrm{i}$ & $8.47 \mathrm{e}-001$ & $1.32 \mathrm{e}+000$ \\
\hline$-1.12 \mathrm{e}+000-6.99 \mathrm{e}-001 \mathrm{i}$ & $8.47 \mathrm{e}-001$ & $1.32 \mathrm{e}+000$ \\
\hline$-1.47 \mathrm{e}+000$ & $1.00 \mathrm{e}+000$ & $1.47 \mathrm{e}+000$ \\
\hline
\end{tabular}

Table 4.1: Reference Dynamics Eigenvalues

In essence, the reference model dynamics were chosen to represent the baseline closed-loop system operating under the baseline LQR controller. Figure 4.2 shows the baseline system response to a series of commanded pitch rate doublets, along with the elevator deflection and rate that were required by the LQR controller, while tracking the pitch rate doublets, without system uncertainties. As seen from the figure, the elevator deflection and rate are well within acceptable limits.
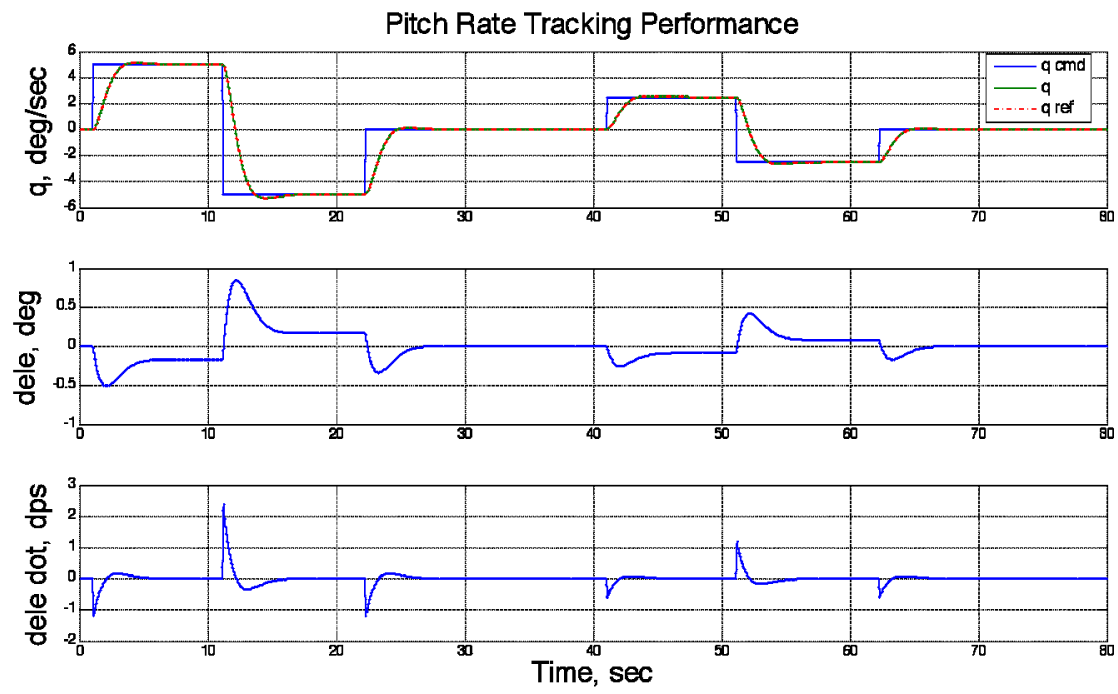

Figure 4.2: Baseline Closed-Loop Response to Pitch Rate Doublets without Uncertainties

With only the baseline controller operating and with uncertainties turned on, closed-loop system tracking performance degradation can be clearly observed from the data that are shown in Figure 4.3. However, both the control input and its rate remain small. 

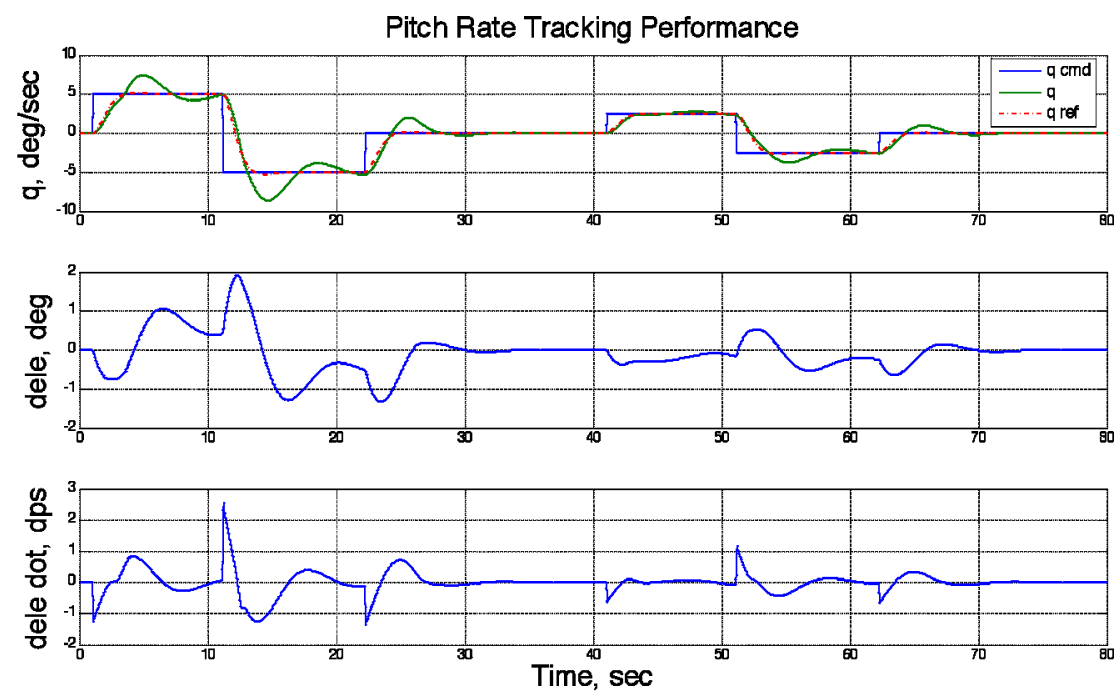

Figure 4.3: Baseline Closed-Loop Response in the Presence of Matched Uncertainties

Next, conventional MRAC laws were constructed by solving algebraic Lyapunov equation (2.18) with

$$
Q_{\text {ref }}=\operatorname{diag}\left(\left[\begin{array}{lll}
0.1 & 1 & 800
\end{array}\right]\right)
$$

Rates of adaptation were chosen as

$$
\Gamma_{x}=\operatorname{diag}\left(\left[\begin{array}{lll}
1 & 400 & 400
\end{array}\right]\right), \quad \Gamma_{\Theta_{d}}=20
$$

and the total control (elevator deflection) was formed as a sum of the baseline LQR controller and its conventional MRAC adaptive augmentation,

$$
\delta_{e}=\delta_{e B L}+\delta_{e A D}
$$

where the adaptive component $\delta_{e A D}$ was defined according to (2.12). We immediately note that since initial conditions in the adaptive laws (2.17) can be chosen arbitrarily, the selected adaptive augmentation architecture is equivalent to a pure adaptive (no baseline included) controller, whose initial conditions $\hat{K}_{x}(0)$ are set equal to the LQR baseline feedback gains. In summary, the total elevator deflection is given by:

$$
\delta_{e}=\left(K_{x B L}+\hat{K}_{x}\right)^{T} x-\hat{\Theta}_{d}^{T} \Phi_{d}(\alpha)
$$

With LQR and MRAC controllers operating simultaneously, the reference / baseline closed-loop system tracking performance is recovered, with acceptable elevator deflection and rates. The data are shown in Figure 4.4.
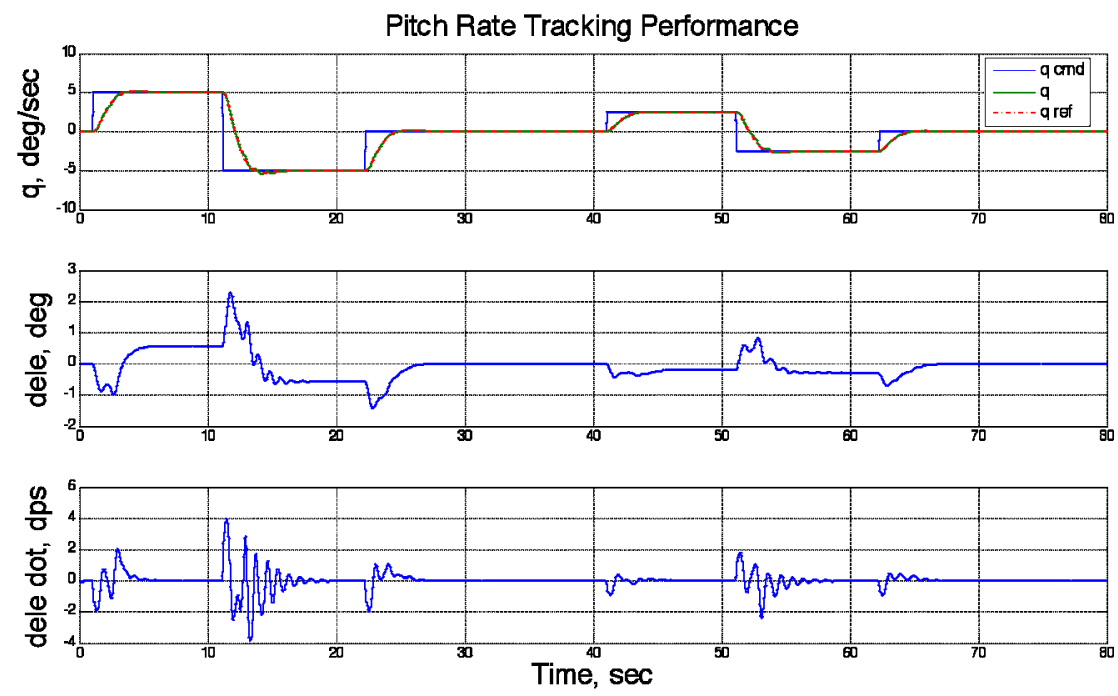
Figure 4.4: (LQR + MRAC) Closed-Loop Response in the Presence of Uncertainties

To demonstrate benefits of PMRAC on the system transient response and control input, PMRAC laws (3.11) were constructed using adaptive rates (4.14) and employing identical RBF-s to the conventional MRAC laws. Rather than selecting matrix $Q_{p r d}$ in (3.5), we use the insight into the physical system present in the selection of $P_{\text {ref }}$ and note that the predictor dynamics should be faster than the reference dynamics. While still satisfying the positive definite requirement of (3.5), we select:

$$
A_{p r d}=3 A_{r e f}, \quad P_{p r d}=3 P_{r e f}
$$

With the LQR and PMRAC controllers turned on, the reference / baseline closed-loop system tracking performance is recovered once again, but with significantly smoother control input than the conventional MRAC design required. The response data are shown in Figure 4.5.
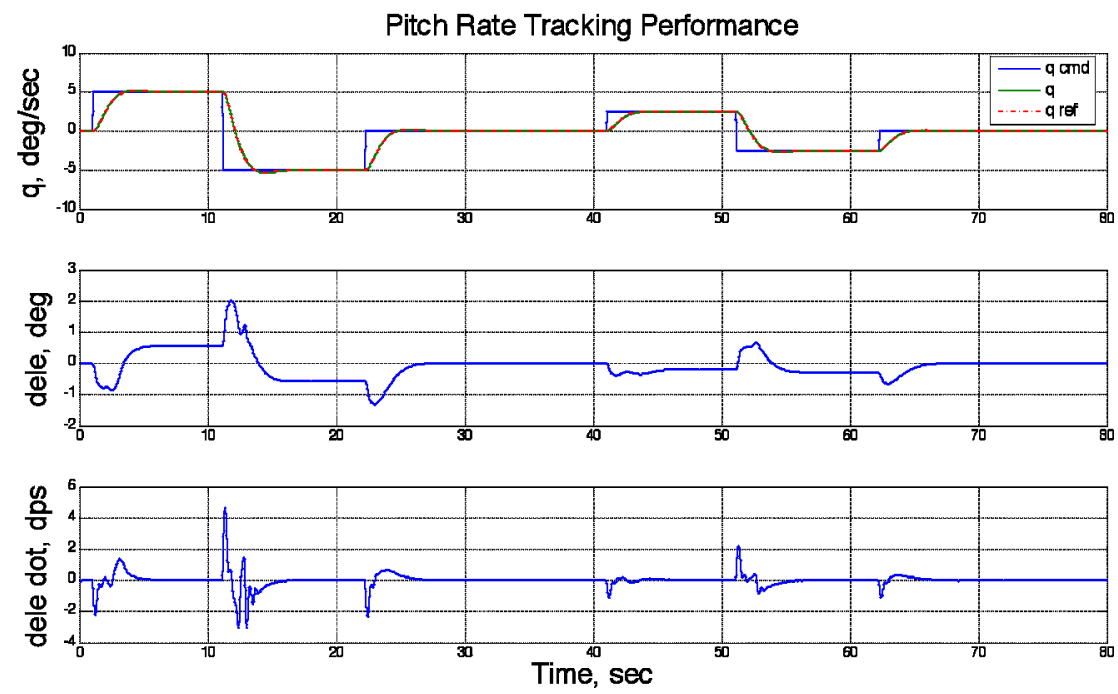

Figure 4.5: (LQR + PMRAC) Closed-Loop Response in the Presence of Uncertainties

The (LQR + MRAC) and (LQR + PMRAC) controllers resulted in comparable tracking performance (Figure 4.4 vs. Figure 4.5), yet the required elevator deflection and its rate were of higher levels and contained unwanted high frequency oscillations in the case of the (LQR + MRAC) design. To better illustrate the advantage of the PMRAC controller, we overlaid the (LQR + MRAC) and the (LQR + PMRAC) tracking responses, both in the presence of the system uncertainties. Figure 4.6 shows the data.

Pitch Rate Tracking Performance
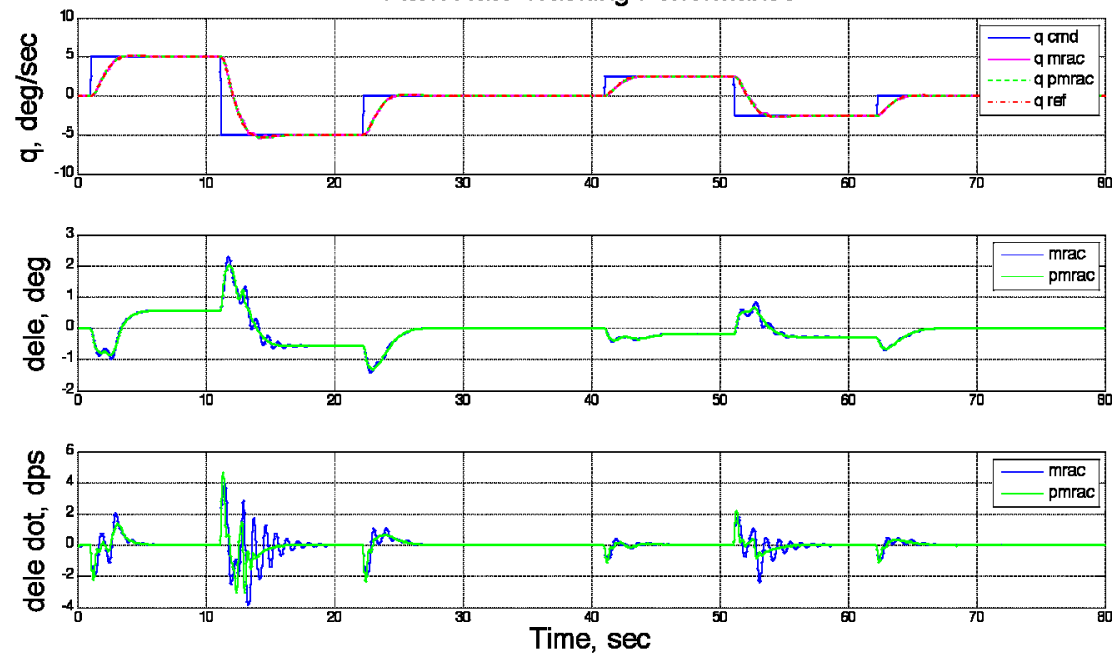

Figure 4.6: Comparison of (LQR + MRAC) vs. (LQR + PMRAC) Closed-Loop Responses 
An alternative way of quantifying control input activity is to use the frequency domain, and to plot their corresponding FFT coefficients. Figure 4.7 shows significantly reduced control input activity in the PMRAC augmentation design.

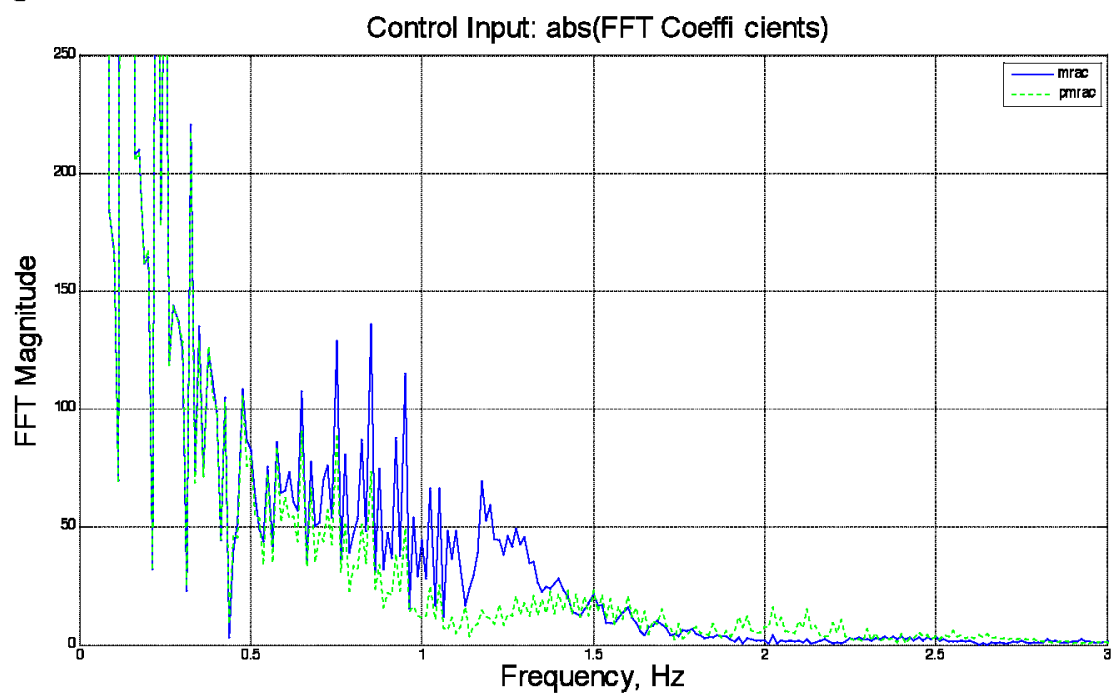

Figure 4.7: Comparison of (LQR + MRAC) vs. (LQR + PMRAC) Control Input FFT Coefficients

The data confirm the fact that that the proposed PMRAC controller provides improved transient response (when compared to MRAC) of the closed-loop system, in the presence of uncertainties. A formal proof of this phenomenon remains an open problem.

As discussed earlier, the better transient performance characteristics provided by PMRAC can be attributed to the inclusion of the prediction error $\hat{e}$ into the adaptive laws. The main difference between a conventional MRAC augmentation and the proposed predictor-based MRAC augmentation lies in the fact that while the parameters in the conventional MRAC are updated based on the tracking error between the plant and the reference model, in the PMRAC case the adaptive laws are also designed to minimize the prediction error in the input-output measurements. In addition to the tracking error in conventional MRAC laws, the prediction error in PMRAC provides an extra information about the system uncertain parameters. This allows the predictor-based adaptive augmentation to retain stability and tracking performance, while potentially improving the robustness and transient performance of a conventional direct MRAC augmentation.

\section{Conclusion}

In this paper, we presented a state-predictor based direct adaptive tracking design methodology for multi-input dynamical systems, with partially known dynamics. Efficiency of the design was demonstrated using short period dynamics of an aircraft. Formal proof of the reported PMRAC benefits constitute future research and will be reported elsewhere.

\section{Acknowledgments and Disclaimer}

This work was sponsored (in part) by NASA grant number NNL06AA04B. The views and conclusions contained herein are those of the authors and should not be interpreted as necessarily representing the official policies or endorsements, either expressed or implied, of NASA or the U.S. Government.

\section{References}

[1] Narendra, K. S., Annaswamy, A. M., Stable Adaptive Systems, Dover, 2005.

[2] Ioannou, P. A., Fidan, B., Adaptive Control Tutorial, SIAM, 2006.

[3] Khalil, H. K., Nonlinear Systems, $3^{\text {rd }}$ ed., Prentice Hall Inc., New York, 2002.

[4] Slotine, J.-J. E., Li, W., Applied Nonlinear Control, Prentice Hall, 1995. 
[5] Pomet, J. B., Praly, L., "Adaptive nonlinear regulation: Estimation from the Lyapunov equation," IEEE Trans. Autom. Control, 37(6), pp.729-740, 1992.

[6] Stevens, B., Lewis, F., Aircraft Control and Simulation, Wiley, New York, 1992. 